\title{
Microarray Analysis of bacterial blight resistance 1 mutant rice infected with Xanthomonas oryzae pv. oryzae
}

\author{
So Young $\mathrm{Yi}^{1 \dagger}$, Ha Yeon Lee ${ }^{1 \dagger}$, Hyun A Kim${ }^{1}$, Chan Ju Lim¹, Woong Bom Kim¹, Hyun A Jang', Jong-Seong Jeon ${ }^{2}$, \\ Suk-Yoon Kwon ${ }^{1 *}$ \\ ${ }^{1}$ Plant Systems Engineering Research Center, KRIBB, 125 Gwahak-ro, Yuseong-gu, Daejeon 305-806, Republic of Korea \\ ${ }^{2}$ Crop Biotech Institute and Graduate School of Biotechnology, Kyung Hee University, Yongin 446-701, Korea
}

\begin{abstract}
We analyzed the transcriptional profile of the Xoo infected $b b r l$ mutant using a commercial rice gene chip containing 51,279 transcripts. Microarray revealed 92 genes with increased levels of expression and 22 genes with decreased levels of expression in $b b r l$. Some of the differentially expressed genes were validated by qRT-PCR. Higher expression of defense-related genes and AP2 domain containing transcription factors along with lower expression of reactive oxygen scavenging enzymes may be responsible for defense signaling in the $b b r 1$ upon Xoo infection. The putative target genes of AP2 domain containing transcription factors also showed differential gene expression during Xoo infection, some of which encoded bacterial pathogen resistance-related protein. Induction of AP2 domain containing transcription factors along with up-regulation of their putative target genes during Xoo infection may inhibit pathogen spread in the $b b r l$. This observation supports the hypothesis that AP2 domain containing transcription factors is involved in the regulation of differentially expressed genes in bbrl.
\end{abstract}

Keywords Bacterial blight resistant rice, Gamma-ray mutant, Xanthomonas oryzae pv. oryzae, ERF and DREB/CBF

\section{INTRODUCTION}

Rice (Oryza sativa L.) is the most important crop in the world as it feeds half of the world's population. The stable production of rice is affected by biotic and abiotic stress. The rice diseases caused by plant pathogenic fungi, bacteria and viruses are capable of causing heavy loss on rice crop, the global yields of which is annually decreasing by $10-15 \%$ (Dai et al. 2007). The use of resistant cultivars is one of the most important factors used to control diseases.

Because worldwide rice production has been severely affected by bacterial blight (BB) caused by Xanthomonas oryzae pv. oryzae (Xoo), early research efforts have been focused on the utilizing disease resistance $(R)$ genes in rice. Since the first cloning of the $R$ gene $X a 21$ which confers resistance to Xoo almost twenty years ago (Song et al. 1995 ), nearly 30 major $R$ genes for resistance to Xoo have been identified (Kurata and Yamazaki 2006) and five of them: Xa1,Xa3/Xa26, xa5, xa13 and Xa27, have been cloned: (Yoshimura et al. 1998; Iyer and McCouch 2004; Sun et al. 2004; Gu et al. 2005; Chu et al. 2006; Jiang et al. 2006). Genetic studies of $\mathrm{BB}$ resistance have resulted in the development of donor lines carrying major $R$ genes. A number of these donor lines have been used in rice breeding programs around the world. Using transgenic approach, agronomically important cultivars such as IR64 and IR72 have been transformed with the $X a 21$ gene and field trials of selected lines were successfully undertaken in China (Zhang et al. 1998; Tu et al. 2000). Further, the Xa21 gene has been introduced into a widely used restorer of hybrid rice in China, Minghui 63, in order to produce BB-resistant hybrid rice with elite agronomic characters (Zhai et al. 2002).

Mutation breeding is also an important tool for crop improvement. During the past 75 years, more than 3,100

Received November 7, 2013; Revised November 25, 2013; Accepted November 27, 2013; Published December 31, 2013

*Corresponding author Suk-Yun Kwon, sykwon@kribb.re.kr, Tel: +82-42-860-4340, Fax: +82-42-860-4349

${ }^{\dagger}$ They are equally contributing authors. 
mutant varieties have been produced worldwide (The FAO/IAEA Mutant Varieties Database, http://mvgs.iaea. org/AboutMutantVarities.aspx). More than 501 new varieties in rice have been obtained by applying different mutagenic agents. In Asia Pacific, there are approximately 343 mutants of rice released (Ahloowalia et al. 2004).

DNA microarray can measure the individual transcript level of tens of thousands of genes simultaneously, thus providing a high-throughput means for analyzing gene expression levels at the whole-genome scale (Schena et al. 1995; Chu et al. 1998) that may help elucidate the network of defense response. For example, microarray has been used to characterize the rice-pathogen interaction, such as the interaction of rice-Xoo (Li et al. 2006; Kottapalli et al. 2007), -flagellin (Fujiwara et al. 2004), -lipopolysaccharides (Desaki et al. 2006), -fungal elicitor (Kim et al. 2005), -rice dwarf virus (Shimizu et al. 2007) and -plant hopper (Cho et al. 2005).

In previous study, six rice mutant lines (M5 generation); TILL300-534, TILL300-537, TILL300-1212, TILL300-793, TILL300-693 and TILL300-651, which present resistant phenotype against Xoo KXO85 were selected from 3000 lines of gamma-ray mutated M3 plants (Lim et al. 2010). Among selected six mutant lines TILL300-651 (Bacterial Blight Resistance $1 ; b b r l)$ showed relatively strong induction of rice $R$ gene $X a 21$ which confers resistance to $X o o$ and other rice $R$ genes such as $P i 36$ and Pi-ta against Magnaporthe oryzae ( $\operatorname{Lim}$ et al. 2010). To further understand defense response in the bbrl-Xoo interaction, we profiled the expression of rice genes involved in the phenotype of rice mutant, $b b r l$, using a commercial rice gene chip containing 51,279 transcripts representing two rice cultivars, indica and japonica.

\section{MATERIALS AND METHODS}

\section{Plant materials and pathogen inoculation}

'Dongan' seeds (Oryza sativa L. cv. japonica) were allowed to imbibe water overnight at $22^{\circ} \mathrm{C}$ and kept on moistened filter paper until germination. Germinated seeds were grown in soil in a greenhouse for 40 days prior to $\mathrm{XoO}$ inoculation.
Bacterial cells of Xoo were suspended in sterile distilled water and the inoculation concentration was adjusted to $\mathrm{OD}_{600}=1.0$ (Song et al. 1995). Rice leaves were inoculated with the bacterial suspension using the leaf clipping method (Kauffman et al. 1973). For gene expression analysis, leaf samples were collected from untreated control and infected leaves at 10 days after inoculation. The samples were flash frozen in liquid nitrogen and stored at $-80^{\circ} \mathrm{C}$

\section{RNA Isolation and qRT-PCR analysis}

Total RNA was isolated from the collected samples using RNaesy mini kit (Qiagen) according to the manufacturer's instruction. Approximately $1 \mu \mathrm{g}$ DNA-free RNA was used for first-strand cDNA synthesis using the Moloney Murine Leukemia Virus (M-MuLV) reverse transcriptase for quantitative real-time polymerase chain reaction (qRT-PCR; Fermentas). The qRT-PCR reactions were performed using a Thermal Cycler Dice Real Time System TP850 (TaKaRa, http://www.takara-bio.com) and SYBR Premix Ex Taq (TaKaRa). Primer sets prepared at $0.1 \mu \mathrm{M}$ final concentration were used for a final volume of $25 \mu \mathrm{L}$. The thermal profile of the qRT-PCR reactions was $10 \mathrm{~min}$ at $95^{\circ} \mathrm{C}, 40$ cycles of $5 \mathrm{~s}$ at $95^{\circ} \mathrm{C} / 20 \mathrm{~s}$ at $60^{\circ} \mathrm{C}$. Subsequently, a dissociation curve was generated. All reactions were carried out in triplicate. Primers used for qRT-PCR are listed in the Table 1.

\section{Microarray analysis}

The microarray experiment was conducted by the DNA Link Corporation (Seoul, Korea) according to the Affymetrix technical manual (http://www.affymetrix. com/support/index.affx). Total RNAs were extracted from the samples stored at -80 ${ }^{\circ} \mathrm{C}$ and gene expression was compared between the wild type 'Dongan' and bbrl mutant with two replicates. Hybridization, washing, staining and scanning procedures were performed as described in the Affymetrix technical manual. The analysis software was the Affymetrix Command Console, R affy-package (2.9.2), Expression Console1.1, DAVID. 
Table 1. Summary table displaying Arabidopsis orthologous of differentially expressed genes with known roles in disease resistance (R- ), transcript regulation (TF- ) and oxidative stress (POD- ) and sequences of forward and reverse primers used in quantitative RT-PCR to validate the 17 selected gene expression changes determined by microarray analysis. TF, Transcription factor; R, Resistance; POD, Peroxidase.

\begin{tabular}{ccccc}
\hline \hline Primer name & Gene title & $\begin{array}{l}\text { Arabidopsis } \\
\text { orthologous }\end{array}$ & Forward-primer & Reverset-primer \\
\hline TF-1 & Os02g0527300 & AT2G26150 & gtggcactagtcagcaagca & tactctcccaagctgcgttt \\
TF-2 & Os06g0127100 & AT4G25480 & ctacgcgtactacggcaaca & gaggagcaaagctggttgag \\
TF-3 & Os08g0474000 & AT4G34410 & gagacaggggaccagctct & ttcaattagtacaccagagccaat \\
TF-4 & Os04g0583900 & AT5G37260 & ctccacaaaacagggagtgg & tgtttttttagctcgcctgt \\
TF-5 & Os03g0327800 & AT3G04070 & ctaggtcgtccgatcatgc & ccggctttatgatcttgaca \\
TF-6 & Os07g0558100 & AT4G21440 & gcaacaaccacaacgtcaac & agtgttcgattcggctctgt \\
TF-7 & Os01g0975300 & AT5G59780 & cagccagaggatgagtcgt & gcgaataatccgagcagaag \\
TF-8 & Os01g0141000 & AT1G13260 & atcagcgtactcctgccta & tgcaatctctgacctgacaaa \\
R-1 & Os01g0944900 & AT4G16260 & gcttactacceggacgtcaa & atgacggatgggttggtg \\
R-2 & Os02g0194700 & AT3G45140 & gctgcatttgggacaagatt & atccgtccgcatgacatact \\
R-3 & Os03g0129100 & AT2G39200 & aaagggtgaggtcggaagat & ggccatcaccgttgtacact \\
R-4 & Os06g0698300 & AT4G31750 & ctgcaagaagctcctccag & tctgcttggcacaagacaac \\
R-5 & Os10g0490800 & AT2G15130 & gtggactacgcgaagcaggt & gtcacctccgtcctcacg \\
POD-1 & Os03g0235000 & AT5G06720 & ggcaactccatggtcaagat & gcgctccacaacacattaaa \\
POD-2 & Os07g0677100 & AT5G05340 & atcaggcttagctgctccaa & tcggtacataacatgggcttc \\
POD-3 & Os07g0677200 & AT5G05340 & agctgctccaaggtgaactc & atggctgctctgctccatac \\
POD-4 & Os08g0113000 & AT4G33420 & ctgaattgccegccttag & cctccatgccacaatacaaa \\
ACTIN & Os03g50890 & AT3G18780 & ggaactggataggtcaaggc & agtctcatggatacccgcag \\
\hline
\end{tabular}

\section{RESULTS AND DISCUSSION}

\section{Isolation of the bbr1 mutant with enhanced Xoo resistance}

To examine whether the Xoo resistant phenotype can be maintained in the next generation, progeny from the TILL300-651 (M5) were tested for the Xoo resistance phenotype. Figure 1a shows a picture of typical leaves from each of the following inoculated wild type 'Dongan' and bbrl mutant. While all bbrl mutant progenies tested were resistant, showing relatively short lesions, inoculated leaves of wild type 'Dongan' developed water soaked long lesions (Fig. 1a, b). This result demonstrates that the bbrl mutant enhanced resistance to Xoo.

\section{Differential gene expression in the leaves of $b b r 1$ mutant plants}

Leaf samples were collected from the uninoculated (no treatment; NT) and Xoo-inoculated (10 DAI) wild type 'Dongan' (WT) and $b b r 1$ mutant (M6), to detect differential gene expression under both conditions. To reduce experimental variation we separately pooled the leaf samples from eight inoculated/uninoculated WT and bbrl mutant of individual biological replicate. Total RNA was then isolated from pooled samples and used for labeling. To identify the significantly differential expression of Xoo responsive genes in bbrl compared to WT, we applied the following criteria; (a) the gene expression change occurred at the same direction (increase or decrease) in replication; and (b) the average ratio (fold change of $b b r 1 /$ WT signal intensity) of expression levels in all microarray analyses was greater than 4 or less than -4. Applying these criteria, we detected 92 genes with increased (up-regulated) levels of expression and 22 genes with decreased (down-regulated) levels of expression in bbrl (Fig. 2). These genes were considered as characteristics of the bbrl mutant during Xoo infection. 
(a)

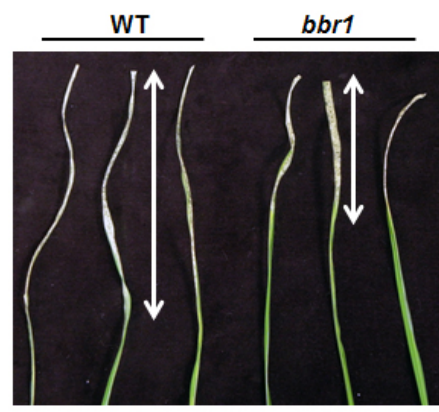

(b)

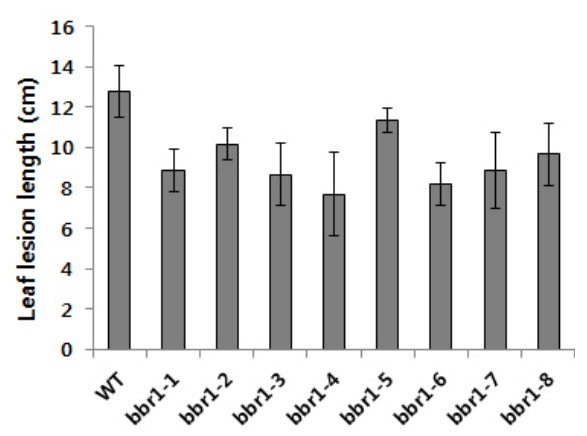

Fig. 1. Phenotypic analysis of the rice bbrl mutant. (a) Water-soaked disease lesions on three leaves from wild type 'Dongan' (WT) and bbrl mutants (M6). 40-day-old plants were inoculated with Xoo. Image was taken two weeks after Xoo inoculation. (b) Leaf lesion lengths of eight bbrl M6 progeny. Lesion lengths were measured at two weeks post Xoo inoculation. Experiments were repeated two times with similar results. Each data point represents the average and standard deviation of at least three leaves.

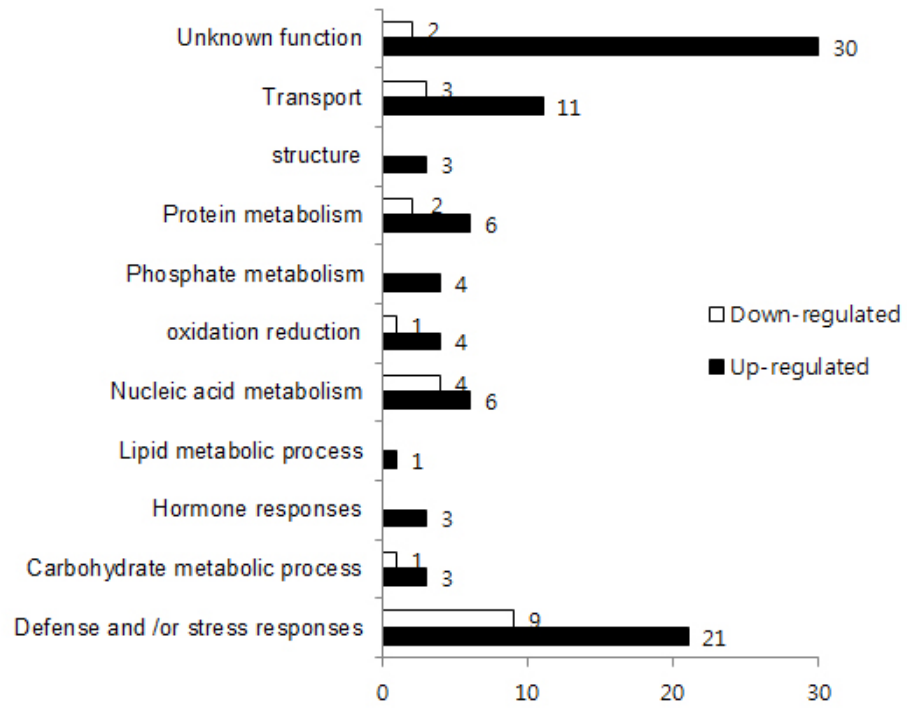

Fig. 2. Gene ontology classification of differentially expressed genes in rice leaves at 10 day post inoculation. Functional categories are derived from the primary annotation of biological process retrieved from the Kyoto Encyclopedia of Genes and Genomes (KEGG; www.genome.jp/kegg). Differentially-regulated genes were expressed at 4-fold higher or lower levels in the $b b r l$ mutant compared to wild type from two independent microarray analyses. White bars indicated the number of four-fold down regulated genes and black bars indicated the number of four-fold up-regulated genes compared to the WT. 
These 114 differentially expressed genes (DEGs) were further analyzed and annotated using public access databases (http://www.genome.jp/kegg). According to predicted functions of their homologous genes in Arabidopsis, these genes could be assigned to eleven biological process categories (Fig. 2), including defense and/or stress response, carbohydrate metabolic process, hormone responses, lipid metabolic process, nucleic acid metabolism, oxidation reduction, phosphate metabolism, protein metabolism, structure, transport and unknown function. Major categories

Table 2. Highlight of differentially expressed genes with known or putative roles in stress response and transcript regulation. Differentially-regulated genes were expressed at 4-fold higher or lower levels in the bbrl mutant compared to wild type from two independent microarray analyses.

\begin{tabular}{|c|c|c|}
\hline Description & Gene Title & Average fold change $^{\mathrm{a}}$ \\
\hline \multicolumn{3}{|l|}{ Transcription factor activity } \\
\hline TF-1; Similar to Heat shock transcription factor 31 & Os02g0527300 & 11.52 \\
\hline TF-2; AP2, Similar to CBF-like protein & Os06g0127100 & 43.92 \\
\hline TF-3; AP2, Similar to AP2 domain containing protein RAP2.6 & Os08g0474000 & 51.32 \\
\hline TF-4; MYB, Similar to LHY protein & Os04g0583900 & 6.12 \\
\hline TF-5; NAM, No apical meristem (NAM) domain containing protein & Os03g0327800 & 5.37 \\
\hline TF-6; MYB, Similar to Myb-related transcription factor LBM1 & Os07g0558100 & 6.04 \\
\hline TF-7; MYB, Similar to Typical P-type R2R3 Myb protein & Os01g0975300 & -7.52 \\
\hline TF-8; AP2, RAV-like protein & Os01g0141000 & -4.17 \\
\hline \multicolumn{3}{|l|}{ Response to biotic stress } \\
\hline R-1; Similar to Beta-1,3-glucanase-like protein & Os01g0944900 & 19.29 \\
\hline R-2; Similar to Lipoxygenase 2.3, chloroplast precursor & Os02g0194700 & 10.9 \\
\hline R-3; Seven transmembrane protein $\mathrm{MLO} 2$ & Os03g0129100 & 8.27 \\
\hline R-4; Protein phosphatase $2 \mathrm{C}$ family protein & Os06g0698300 & 7 \\
\hline R-5; Similar to NtPRp27 & Os10g0490800 & 14.22 \\
\hline Similar to Iron-phytosiderophore transporter protein yellow stripe 1 & Os02g0649900 & -6.28 \\
\hline Similar to Pathogen-related protein & Os01g0731100 & -8.17 \\
\hline \multicolumn{3}{|l|}{ Response to oxidative stress } \\
\hline POD-1; Peroxidase & Os03g0235000 & -42.2 \\
\hline POD-2; Peroxidase & Os07g0677100 & -7.73 \\
\hline POD-3; Peroxidase & Os07g0677200 & -4.79 \\
\hline POD-4; Similar to Peroxidase 47 precursor & Os08g0113000 & -14.6 \\
\hline \multicolumn{3}{|l|}{ Response to abiotic stress } \\
\hline Late embryogenesis abundant (LEA) group 1 family & Os04g0589800 & 25.25 \\
\hline Similar to Low-temperature induced protein $1 \mathrm{t} 101.2$ & Os05g0122700 & 16.92 \\
\hline Similar to Allyl alcohol dehydrogenase & Os04g0497000 & 11.48 \\
\hline Similar to 1-Cys peroxiredoxin; & Os07g0638300 & 9.16 \\
\hline Similar to Small heat stress protein class CIII & Os02g0782500 & 8.33 \\
\hline GRAM domain containing protein; & Os12g0478100 & 7.53 \\
\hline Similar to Acyl-CoA-binding protein 2 (ACBP 2) & Os06g0115300 & 6.89 \\
\hline Hly-III related proteins family protein & Os06g0652200 & 6.54 \\
\hline Similar to Dehydrin DHN1 (B8) & Os01g0702500 & 6.42 \\
\hline Heat shock protein DnaJ, N-terminal domain containing protein & Os01g0606900 & 6.34 \\
\hline EFA27 for EF hand, abscisic acid, $27 \mathrm{kD}$ & Os04g0511200 & 4.86 \\
\hline Glycoside hydrolase, family 17 protein & Os01g0860800 & 4.44 \\
\hline Similar to germin-like protein 8 & Os08g0189850 & -6.45 \\
\hline Similar to germin-like protein 12 & Os08g0189900 & -6.92 \\
\hline
\end{tabular}

${ }^{a}$ Average values of Xoo inoculated $b b r l$ samples, compared to WT samples, from two independent microarray analysis. Numbers show the factor of change between wild type and mutant after Xoo inoculation; positive values represent up-regulation (e. g. $3=3$-fold increase) negative values down-regulation (e. g. $-3=3$-fold decrease). 
of DEGs were biotic and abiotic stress related and transcription factors (Table 2). Thirty-three genes were classified into these categories and only considered for functions on specific pathways to further illustrate the differential response of WT and bbrl mutant to Xoo infection (Table 2).

\section{Defense-related genes induced in $b b r 1$ mutant}

Higher expression of $\beta$-1,3-glucanase-like protein (Os01g0944900; >19 fold), lipoxygenase 2.3, chloroplast precursor (Os02g0194700; >10 fold), seven transmembrane protein MLO2 (Os03g0129100; >8 fold), protein phosphatase 2C family protein (Os06g0698300; 7 fold) and basic secretory protein $(\mathrm{Os} 10 \mathrm{~g} 0490800 ;>14$ fold) genes were detected at $10 \mathrm{DAI}$ in $b b r 1$ mutant plant. On the other hand, lower expression of iron-phytosiderophore transporter protein yellow stripe 1 (Os02g0649900; >6 fold) and pathogen-related protein (Os01g0731100; $>8$ fold) were detected in Xoo-infected bbrl leaf tissue (Table 1). qRT-PCR analysis further confirmed the expression of

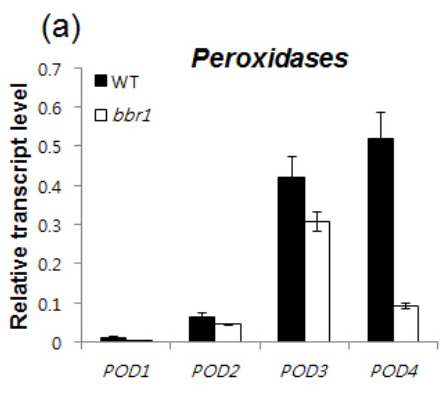

(b)
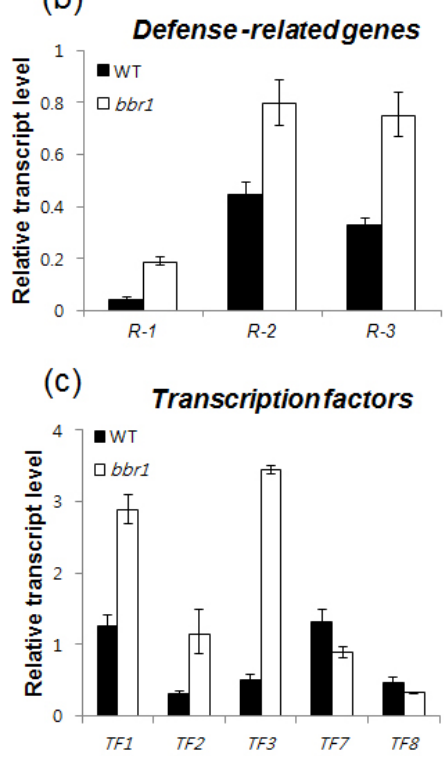

Fig. 3. Altered expressions of genes related to Xoo inoculation in bbrl. The rice ACTIN gene was used as an internal positive control and transcript levels of the tested genes were normalized with that of ACTIN. Relative expressions of the tested genes were compared with that in WT plants. Bar represents standard deviation (three replicates) (a) qRT-PCR analysis was performed to study the transcript levels of peroxidase genes in the leaves of WT and bbr1 plants at the 40-day-old stage. The tested genes include POD-1, Os03g0235000; POD-2, Os07g0677100; $P O D-3$, Os07g0677200; POD-4, Os08g0113000. (b) qRT-PCR analysis of the transcripts level of defense-related genes in the leaves of WT and bbrl plants at the 40-day-old stage. The tested genes include $R-1$, Os01g0944900; $R-2$, Os02g0194700; $R-3$, Os03g0129100. (c) qRT-PCR analysis of the transcripts level of transcription factor genes in the in the leaves of WT and bbrl plants at the 40-day-old stage. The tested genes include $T F-1$, Os02g0527300; TF-2, Os06g0127100; TF-3, Os08g0474000; TF-7, Os01g0975300; TF-8, Os01g0141000. 
representative five genes $(\mathrm{R}-1 \sim \mathrm{R}-5$; Table 1$)$ that were highly up-regulated in bbrl mutant 10 DAI. Among the five genes, three [Os01g0944900 ( $\beta$-1,3-glucanase-like protein; R-1), Os02g0194700 (lipoxygenase 2.3 chloroplast precursor (R-2); and Os03g0129100 (seven transmembrane protein MLO2; R-3) were expressed at higher levels in $b b r l$ than in WT after Xoo inoculation (Fig. 3a).

As a member of the PR-2 group of pathogenesis-related (PR) proteins, $\beta-1,3$-glucanase is induced by pathogen infection and plays an active antifungal role in hydrolyzing B-1,3-glucan, a major structural component of fungal cell walls (Sela-Buurlage et al. 1993; Jach et al. 1995). Lipid peroxidation, triggered by lipoxygenases and reactive oxygen species (ROS), is a hallmark of plant pathogen responses, both in signal transduction processes and during the execution of programmed cell death. Lipoxygenases oxidize free fatty acids in the cytosol or chloroplasts, thereby initiating several oxylipin pathways including the jasmonic acid and hydroperoxide lyase pathway (Mosblech et al. 2009). Twelve predicted Mlo homologs were identified in the rice genome (Goff et al. 2002). The seven transmembrane MLO protein (Devoto et al. 1999) is thought to mediate defense suppression against Blumeria graminis f. sp. hordei attack via direct $\mathrm{Ca}^{2+}$-dependent interaction with calmodulin (Kim et al. 2002). MLOmediated defense suppression also likely involves one or several small GTP-binding proteins of the ROP (Rhorelated GTPases from plants) family (Schultheiss et al. 2002). Consistent with its involvement in plant-microbe interactions, $M L O$ expression is induced upon biotic and abiotic stress stimuli (Piffanelli et al. 2002). Induction of defense related genes during Xoo infection may inhibit pathogen spread in the $b b r 1$.

\section{Peroxidase genes reduced in $b b r 1$ mutant}

Four class III peroxidases genes [Os03g0235000 (POD-1), Os07g0677100 (POD-2), Os07g0677200 (POD-3) and Os08g0113000 (POD-4) were expressed at lower levels in the $b b r l$ mutant than in WT 10 DAI (Table 2). qRT-PCR also confirmed lower level of four peroxidases expression (Fig. 3b). Peroxidases belong to well-known class of PR-9 proteins (van Loon et al. 2006). They are expressed to limit cellular spreading of the infection through the establishment of structural barriers or generation of highly toxic environments by massively producing ROS and reactive nitrogen species (RNS) (Passardi et al. 2005). Since POD scavenges the ROS, a reduced activity of this enzyme will increase the levels of ROS and result in hastened cell death. Down-regulation of PODs in $b b r l$ may therefore result in enhanced resistance against the invading pathogen. A lower expression of four PODs in $b b r l$ mutant implies the ROS involvement in defense against Xoo invasion.

\section{Induced and suppressed genes encoding transcription factors}

WRKY, myeloblastosis (MYB), APETALA2 (AP2) domain containing transcription factors (ethylene-responsive element-binding proteins; EREBPs, C-repeat-binding proteins; $\mathrm{CBFs}$, related to $\mathrm{ABI} / \mathrm{VP} 1$; RAV), basic region/leucine zipper motif (bZIP), no apical meristem (NAM), zincfinger proteins, and heat shock factors (HSFs) are encoded by large gene families and have been intensively studied for their roles in stress responses (Kagaya et al. 1999; Singh et al. 2002; Saibo et al. 2009; Hirayama and Shinozaki 2010; Santos et al. 2011; Scharf et al. 2012).

Higher expression of heat shock transcription factor 31 (Os02g0527300; >11 fold), RAP2.6 (Os08g0474000; >50 fold) and CBF-like protein (Os06g0127100; >40 fold), MYB domain containing protein LHY (Os04g0583900;>6 fold) and LBM1 (Os07g0558100; $>6$ fold) and NAM domain containing protein (Os03g0327800; $>5$ fold) genes were detected in $b b r 1$ mutant plant 10 DAI. On the other hand, lower expression of typical P-type R2R3 MYB protein (Os01g0975300; $>7$ fold) and AP2 domain containing protein RAV (Os01g0141000; $>4$ fold) were detected in Xoo-infected $b b r l$ leaf tissue (Table 2). qRT-PCR analysis further confirmed the expression of eight representative transcription factor genes (TF1-1 TF-8; Table 1) that were highly up- and down-regulated in bbrl mutant 10 DAI. The result showed that expression levels of TF-1 (heat shock transcription factor 31), TF-2 (CBF-like protein) and TF-3 (RAP2.6) were increased in bbrl whereas TF-7 (typical P-type R2R3 Myb protein) and TF-8 (RAV-like protein) were suppressed in $b b r l$ which are in agreement with the microarray results (Fig. 3c). Due to the central role of ERF and CBFs/DREBs in biotic and abiotic 
stress responses and their ability to regulate a large number of stress-responsive target genes, induction of these transcription factors are involved in the regulation of differentially expressed genes in $b b r 1$.

\section{AP2 domain containing transcription factors is involved in the regulation of differentially expressed genes in bbr1}

On the basis of the number of AP2/ERF domains encoded and the gene function, the AP2/EREBP gene family has been divided into four subfamilies: AP2, RAV, DREB and ERF (Sakuma et al. 2002). Both ERF and DREB/CBF subfamilies are of particular interest owing to their involvement in plant responses to stresses. $E R F$ subfamily genes encode a large number of ERFs (Fujimoto et al. 2000), which have been shown to participate in the plant response to biotic stress such as pathogens by recognizing the cis-acting element GCCGCC, known as the GCC-box (Hao et al. 1998). The DREB subfamily genes play an important role in the resistance of plants to abiotic stresses by recognizing the dehydration responsive element (DRE), which has a core motif CCGAC (Liu et al. 1998). The transcription factors, RAV1 and RAV2, contain an AP2 domain in the $\mathrm{N}$-terminal regions and a B3 domain in the C-terminal regions (Kagaya et al. 1999). Using binding site selection assays, the AP2 and $\mathrm{B} 3$ domains of RAV1 were found to bind to the CAACA and CACCTG motifs (Kagaya et al. 1999). Ectopic expression of the
CaRAVI gene in transgenic Arabidopsis plants resulted in the induction of some pathogenesis related (PR) genes, enhancing resistance against infection by bacterial pathogens, and tolerance to osmotic stresses caused by high salinity and dehydration conditions (Sohn et al. 2006).

Among the differentially expressed transcription factor genes observed in this study, the average expression fold change of RAP2.6 (51.32 fold higher than wild type) and CBF like protein (43.92 fold higher than wild type) are the highest in the bbrl. Another AP2 domain containing transcription factor RAV-like protein (4.17 fold lower than wild type) is also suppressed in the $b b r l$ after Xoo infection (Table 2). The extent of induction or repression of an individual gene by Xoo infection in bbrl depends on a complex interaction between its transcription apparatus and associated regulation related sequences. To analyze the promoter region of target genes we selected 117 candidates which are more than two fold up- or down-regulated genes in $b b r l$ and used sequences located $2 \mathrm{~kb}$ upstream of the 5' termini of each candidate genes. Among 117 candidate genes, GCC-box (GCCGCC; Table S1), DRE-related core motifs (CCGAC; Table S2) and AP2+B3 binding motif (CAACA and CACCTG; Table S3) are found in the promoter regions of $90(73.77 \%)$ putative target genes (Fig. 4). Among the genes we detected, several were reported to be related to disease resistance, such as F-BOX STRESS INDUCED 2 (Maldonado-Calderon et al. 2012), PEN3 (Xin et al. 2013), RPM1-INDUCED PROTEIN KINASE

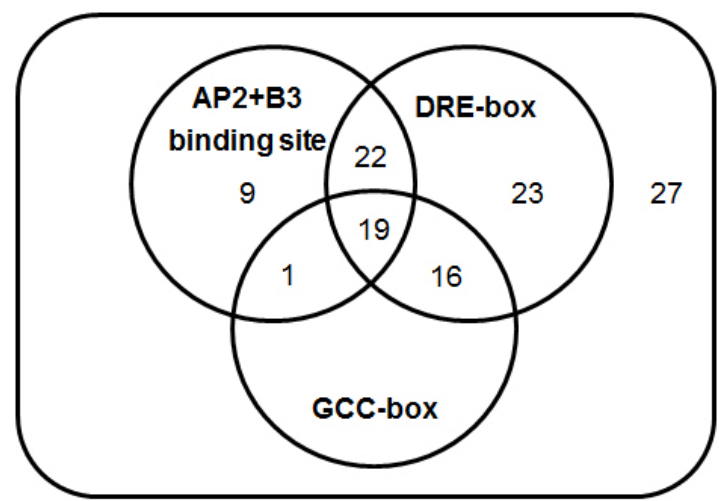

Fig. 4. Venn diagram of more than two folds up- and down-regulated genes with different AP2 domain binding motif in $b b r l$ mutant. A total 117 genes were differentially expressed before and after Xoo infection, among which 90 putative target genes contain DRE-related core motifs (80 genes), GCC-box (36 genes) and AP2+B3 binding motif (51 genes) in their putative promoter regions. 
(Feng et al. 2012), WRKY108 (Higashi et al. 2008) and BETA-1,3-GLUCANASE 2 (Silipo et al. 2005). This observation supports the hypothesis that AP2 domain containing transcription factors are involved in the regulation of differentially expressed genes in $b b r l$.

\section{CONCLUSION}

Japonica rice mutant $b b r l$ which is selected by mutant screening in this study was characterized. The up- and down-regulated genes identified in Xoo resistant $b b r 1$ may play a role in resistance. To investigate the Xoo resistance of the gamma-ray irradiated mutant line, $b b r l$, we performed the commercial rice gene chip analysis. Through DNA microarray analysis, we found significantly elevated expression of AP2 domain containing transcription factor genes, RAP2.6 (Os08g0474000) and $C B F(\mathrm{Os} 06 \mathrm{~g} 0127100)$. Because the primary role of ERF and CBFs/DREBs is the regulation of a large number of target stress-responsive genes in biotic and abiotic stress, the enriched expression of the transcription factors suggests that they might be involved in the regulation of differentially expressed genes in bbrl. Also, the suppression of ROS scavenging enzyme, class III POD genes (Os03g023500, Os07g0677100, Os07g0677200, and Os08g0113000) was detected from DNA microarray analysis of $b b r l$. Down-regulation of PODs in $b b r l$ may contribute to the resistance against the invading pathogen. A lower expression of four PODs in bbrl mutant than wild-type implies that increased ROS may have influence on the defense against Xoo invasion. These observations support the hypothesis that the genes mentioned above, contribute to bacterial blight resistance in bbrl mutant.

\section{ACKNOWLEDGMENTS}

This work was supported by grants from the Technology Development Program for Agriculture and Forestry, Ministry of Agriculture, Food and Rural Affairs, Republic of Korea, and the KRIBB Research Initiative Program.

\section{REFERENCES}

Ahloowalia BS, Maluszynski M, Nichterlein K. 2004. Global impact of mutation-derived varieties. Euphytica 135: $187-204$.

Cho SK, Jung KW, Jeung JU, Kang KH, Shim KS, You MK, Yoo KS, Ok SH, Shin JS. 2005. Analysis of differentially expressed transcripts from planthopperinfested wild rice (Oryza minuta). Plant Cell Rep. 24: 59-67.

Chu S, DeRisi J, Eisen M, Mulholland J, Botstein D, Brown PO, Herskowitz I. 1998. The transcriptional program of sporulation in budding yeast. Science. 282: 699-705.

Chu Z, Yuan M, Yao J, Ge X, Yuan B, Xu C, Li X, Fu B, Li Z, Bennetzen JL, Zhang Q, Wang S. 2006. Promoter mutations of an essential gene for pollen development result in disease resistance in rice. Genes Dev. 20: 1250-1255.

Dai LY, Liu XL, Xiao YH, Wang GL. 2007. Recent advances in cloning and characterization of disease resistance genes in rice. J. Integr. Plant Biol. 49: 112-119.

Desaki Y, Miya A, Venkatesh B, Tsuyumu S, Yamane H, Kaku H, Minami E, Shibuya N. 2006. Bacterial lipopolysaccharides induce defense responses associated with programmed cell death in rice cells. Plant Cell Physiol. 47: 1530-1540.

Devoto A, Piffanelli P, Nilsson I, Wallin E, Panstruga R, von Heijne G, Schulze-Lefert P. 1999. Topology, subcellular localization, and sequence diversity of the Mlo family in plants. J. Biol. Chem. 274: 34993-35004.

Feng F, Yang F, Rong W, Wu X, Zhang J, Chen S, He C, Zhou JM. 2012. A Xanthomonas uridine 5'-monophosphate transferase inhibits plant immune kinases. Nature 485: 114-118.

Fujimoto SY, Ohta M, Usui A, Shinshi H, Ohme-Takagi M. 2000. Arabidopsis ethylene-responsive element binding factors act as transcriptional activators or repressors of GCC box-mediated gene expression. Plant Cell 12: 393-404.

Fujiwara S, Tanaka N, Kaneda T, Takayama S, Isogai A, Che FS 2004. Rice cDNA microarray-based gene expression profiling of the response to flagellin perception in cultured rice cells. Mol. Plant-Microbe Interact. 17: 
986-998.

Goff SA, Ricke D, Lan TH, Presting G, Wang R, Dunn M, Glazebrook J, Sessions A, Oeller P, Varma H, Hadley D, Hutchison D, Martin C, Katagiri F, Lange BM, Moughamer T, Xia Y, Budworth P, Zhong J, Miguel T, Paszkowski U, Zhang S, Colbert M, Sun WL, Chen L, Cooper B, Park S, Wood TC, Mao L, Quail P, Wing R, Dean R, Yu Y, Zharkikh A, Shen $\mathrm{R}$, Sahasrabudhe $\mathrm{S}$, Thomas A, Cannings R, Gutin A, Pruss D, Reid J, Tavtigian S, Mitchell J, Eldredge G, Scholl T, Miller RM, Bhatnagar S, Adey N, Rubano T, Tusneem N, Robinson R, Feldhaus J, Macalma T, Oliphant A, Briggs S. 2002. A draft sequence of the rice genome (Oryza sativa L. ssp. japonica). Science 296: 92-100.

Gu K, Yang B, Tian D, Wu L, Wang D, Sreekala C, Yang F, Chu Z, Wang GL, White FF, Yin Z. 2005. R gene expression induced by a type-III effector triggers disease resistance in rice. Nature 435: 1122-1125.

Hao D, Ohme-Takagi M, Sarai A. 1998. Unique mode of GCC box recognition by the DNA-binding domain of ethylene-responsive element-binding factor (ERF domain) in plant. J. Biol. Chem. 273: 26857-26861.

Higashi K, Ishiga Y, Inagaki Y, Toyoda K, Shiraishi T, Ichinose Y. 2008. Modulation of defense signal transduction by flagellin-induced WRKY41 transcription factor in Arabidopsis thaliana. Mol. Genet. Genomics 279: 303-312.

Hirayama T, Shinozaki K. 2010. Research on plant abiotic stress responses in the post-genome era: past, present and future. Plant J. 61: 1041-1052.

Iyer AS, McCouch SR. 2004. The rice bacterial blight resistance gene xa5 encodes a novel form of disease resistance. Mol Plant Microbe Interact. 17: 1348-1354.

Jach G, Gornhardt B, Mundy J, Logemann J, Pinsdorf E, Leah R, Schell J, Maas C. 1995. Enhanced quantitative resistance against fungal disease by combinatorial expression of different barley antifungal proteins in transgenic tobacco. Plant J. 8: 97-109.

Jiang GH, Xia ZH, Zhou YL, Wan J, Li DY, Chen RS, Zhai WX, Zhu LH. 2006. Testifying the rice bacterial blight resistance gene xa5 by genetic complementation and further analyzing xa5 (Xa5) in comparison with its homolog TFIIAgamma1. Mol. Genet. Genomics 275: 354-366.
Kagaya Y, Ohmiya K, Hattori T. 1999. RAV1, a novel DNA-binding protein, binds to bipartite recognition sequence through two distinct DNA-binding domains uniquely found in higher plants. Nucleic Acids Res. 27: 470-478.

Kauffman HE, Reddy APK, Hsieh SPV, Marca SD. 1973. An improved technique for evaluation of resistance of rice varieties to Xanthomonas oryzae. Plant Dis. Rep. 57: 537-541.

Kim KM, Cho SK, Shin SH, Kim GT, Lee JH, Oh BJ, Kang KH, Hong JC, Choi JY, Shin JS, Chung YS. 2005. Analysis of differentially expressed transcripts of fungal elicitor- and wound-treated wild rice (Oryza grandiglumis). J. Plant Res. 118: 347-354.

Kim MC, Panstruga R, Elliott C, Muller J, Devoto A, Yoon HW, Park HC, Cho MJ, Schulze-Lefert P. 2002. Calmodulin interacts with MLO protein to regulate defence against mildew in barley. Nature 416: 447-451.

Kottapalli KR, Rakwal R, Satoh K, Shibato J, Kottapalli P, Iwahashi H, Kikuchi S. 2007. Transcriptional profiling of indica rice cultivar IET8585 (Ajaya) infected with bacterial leaf blight pathogen Xanthomonas oryzae pv oryzae. Plant Physiol. Biochem. 45: 834-850.

Kurata N, Yamazaki Y. 2006. Oryzabase. An integrated biological and genome information database for rice. Plant Physiol. 140: 12-17.

Li Q, Chen F, Sun L, Zhang Z, Yang Y, He Z. 2006. Expression profiling of rice genes in early defense responses to blast and bacterial blight pathogens using cDNA microarray. Physiological and Mol. Plant Pathol. 68: 51-60.

Lim CJ, Lee HY, Kim WB, Ahmad R, Moon JS, Kim DS, Kwon SY. 2010. Screening of gamma radiation-induced pathogen resistance rice lines against Xanthomonas oryzae pv. oryxae. J. Radiation Ind. 4: 209-213.

Liu Q, Kasuga M, Sakuma Y, Abe H, Miura S, Yamaguchi-Shinozaki K, Shinozaki K. 1998. Two transcription factors, DREB1 and DREB2, with an EREBP/AP2 DNA binding domain separate two cellular signal transduction pathways in drought- and lowtemperature-responsive gene expression, respectively, in Arabidopsis. Plant Cell 10: 1391-1406.

Maldonado-Calderon MT, Sepulveda-Garcia E, Rocha-Sosa M. 2012. Characterization of novel F-box proteins in plants induced by biotic and abiotic stress. Plant Sci. 
185-186: 208-217.

Mosblech A, Feussner I, Heilmann I. 2009. Oxylipins: structurally diverse metabolites from fatty acid oxidation. Plant Physiol. Biochem. 47: 511-517.

Passardi F, Cosio C, Penel C, Dunand C. 2005. Peroxidases have more functions than a Swiss army knife. Plant Cell Rep. 24: 255-265.

Piffanelli P, Zhou F, Casais C, Orme J, Jarosch B, Schaffrath U, Collins NC, Panstruga R, Schulze-Lefert P. 2002. The barley MLO modulator of defense and cell death is responsive to biotic and abiotic stress stimuli. Plant Physiol. 129: 1076-1085.

Saibo NJ, Lourenco T, Oliveira MM. 2009. Transcription factors and regulation of photosynthetic and related metabolism under environmental stresses. Ann. Bot. 103: 609-623.

Sakuma Y, Liu Q, Dubouzet JG, Abe H, Shinozaki K, Yamaguchi-Shinozaki K. 2002. DNA-binding specificity of the ERF/AP2 domain of Arabidopsis DREBs, transcription factors involved in dehydration- and cold-inducible gene expression. Biochem. Biophys. Res. Commun. 290: 998-1009.

Santos AP, Serra T, Figueiredo DD, Barros P, Lourenco T, Chander S, Oliveira MM, Saibo NJ. 2011. Transcription regulation of abiotic stress responses in rice: a combined action of transcription factors and epigenetic mechanisms. OMICS 15: 839-857.

Scharf KD, Berberich T, Ebersberger I, Nover L. 2012. The plant heat stress transcription factor (Hsf) family: structure, function and evolution. Biochim. Biophys. Acta. 1819: 104-119.

Schena M, Shalon D, Davis RW, Brown PO. 1995. Quantitative monitoring of gene expression patterns with a complementary DNA microarray. Science 270: 467-470.

Schultheiss H, Dechert C, Kogel KH, Huckelhoven R. 2002. A small GTP-binding host protein is required for entry of powdery mildew fungus into epidermal cells of barley. Plant Physiol. 128: 1447-1454.

Sela-Buurlage MB, Ponstein AS, Bres-Vloemans SA, Melchers LS, Van Den Elzen P, Cornelissen B. 1993. Only Specific Tobacco (Nicotiana tabacum) Chitinases and [beta]-1,3-Glucanases Exhibit Antifungal Activity. Plant Physiol. 101: 857-863.

Shimizu T, Satoh K, Kikuchi S, Omura T. 2007. The repression of cell wall- and plastid-related genes and the induction of defense-related genes in rice plants infected with Rice dwarf virus. Mol. Plant-Microbe Interact. 20: 247-254.

Silipo A, Molinaro A, Sturiale L, Dow JM, Erbs G, Lanzetta R, Newman MA, Parrilli M. 2005. The elicitation of plant innate immunity by lipooligosaccharide of Xanthomonas campestris. J. Biol. Chem. 280: 33660-33668.

Singh K, Foley RC, Onate-Sanchez L. 2002. Transcription factors in plant defense and stress responses. Curr. Opin. Plant Biol. 5: 430-436.

Sohn KH, Lee SC, Jung HW, Hong JK, Hwang BK. 2006. Expression and functional roles of the pepper pathogeninduced transcription factor RAV1 in bacterial disease resistance, and drought and salt stress tolerance. Plant Mol. Biol. 61: 897-915.

Song WY, Wang GL, Chen LL, Kim HS, Pi LY, Holsten T, Gardner J, Wang B, Zhai WX, Zhu LH, Fauquet C, Ronald P. 1995. A receptor kinase-like protein encoded by the rice disease resistance gene, Xa21. Science 270: 1804-1806.

Sun X, Cao Y, Yang Z, Xu C, Li X, Wang S, Zhang Q. 2004. Xa26, a gene conferring resistance to Xanthomonas oryzae pv. oryzae in rice, encodes an LRR receptor kinase-like protein. Plant J. 37: 517-527.

Tu J, Zhang G, Datta K, Xu C, He Y, Zhang Q, Khush GS, Datta SK. 2000. Field performance of transgenic elite commercial hybrid rice expressing bacillus thuringiensis delta-endotoxin. Natl. Biotechnol. 18: 1101-1104.

van Loon LC, Rep M, Pieterse CM. 2006. Significance of inducible defense-related proteins in infected plants. Annu. Rev. Phytopathol. 44: 135-162.

Xin XF, Nomura K, Underwood W, He SY. 2013. Induction and suppression of PEN3 focal accumulation during Pseudomonas syringae pv. tomato DC3000 infection of Arabidopsis. Mol. Plant-Microbe Interact. 26: 861-867.

Yoshimura S, Yamanouchi U, Katayose Y, Toki S, Wang ZX, Kono I, Kurata N, Yano M, Iwata N, Sasaki T. 1998. Expression of Xa1, a bacterial blight-resistance gene in rice, is induced by bacterial inoculation. Proc. Natl. Acad. Sci. U S A. 95: 1663-1668.

Zhai W, Wang W, Zhou Y, Li X, Zheng X, Zhang Q, 
Wang G, Zhu L. 2002. Breeding bacterial blightresistant hybrid rice with the cloned bacterial blight resistance gene Xa21. Mol. Breed. 8: 285-293.

Zhang S, Song W-Y, Chen L, Ruan D, Taylor N, Ronald
P, Beachy R, Fauquet C. 1998. Transgenic elite Indica rice varieties, resistant to Xanthomonas oryzae pv. oryzae. Mol. Breed.. 4: 551-558. 
Table S1. Thirty-six GCC-box containing differentially expressed genes in bbrl mutant compared to wild type.

\begin{tabular}{|c|c|c|c|c|c|c|c|}
\hline Gene Description & Gene Title & AGI Number & \multicolumn{3}{|c|}{$\begin{array}{c}\text { Number of GCC-box in } \\
\text { putative promoter } \\
\text { region }^{\mathrm{a}}\end{array}$} & \multicolumn{2}{|c|}{$\begin{array}{c}\text { Average } \\
\text { fold change }\end{array}$} \\
\hline \multicolumn{8}{|l|}{ Transcription factor activity } \\
\hline \multicolumn{8}{|l|}{ Response to stress } \\
\hline HSF-type DNA-binding domain containing protein & Os06g0553100 & AT3G24520 & 0 & 4 & 4 & 2.69 & 24.93 \\
\hline VQ domain containing protein & Os01g0278000 & AT2G41010 & 1 & 1 & 2 & 4.84 & 7.29 \\
\hline oxidoreductase/ transition metal ion binding protein & Os09g0445600 & AT5G19875 & 1 & 5 & 6 & 3.57 & 6.87 \\
\hline Similar to F-BOX STRESS INDUCED 2 & Os07g0561300 & AT4G21510 & 5 & 3 & 8 & 3.64 & 6.57 \\
\hline PR (pathogenesis-related) peptide & Os12g0437800 & AT2G38870 & 12 & 5 & 17 & -3.81 & 1.06 \\
\hline \multicolumn{8}{|l|}{ Protein modification process } \\
\hline protein phosphatase $2 \mathrm{C}$ & Os03g0268600 & AT2G29380 & 1 & 2 & 3 & 7.29 & 9.99 \\
\hline STE_MEKK_ste11_MAP3K.6 - STE kinases & Os01g0699500 & AT5G55090 & 2 & 0 & 2 & 9.48 & 9 \\
\hline receptor protein kinase CRINKLY4 precursor & Os08g0374600 & AT3G55950 & 2 & 0 & 2 & 4.94 & 8.88 \\
\hline protein kinase & Os02g0165100 & AT1G16670 & 2 & 0 & 2 & 6.63 & 8.63 \\
\hline STE_MEKK_ste11_MAP3K.4 - STE kinases & Os01g0699100 & AT5G55090 & 2 & 2 & 4 & 3.1 & 7.44 \\
\hline protein phosphatase $2 \mathrm{C}$ & Os01g0583100 & AT1G17550 & 1 & 1 & 2 & 2.93 & 3.77 \\
\hline \multicolumn{8}{|l|}{ Nucleotide binding } \\
\hline WD domain containing protein & Os01g0383700 & AT4G03020 & 2 & 0 & 2 & 4.42 & 5.19 \\
\hline \multicolumn{8}{|l|}{ Metabolic procss } \\
\hline Anthranilate synthase alpha 2 subunit & Os03g0264400 & AT2G29690 & 2 & 0 & 2 & 3.4 & 3.23 \\
\hline starch synthase & Os06g0133000 & AT1G32900 & 1 & 1 & 2 & 13.45 & 1.23 \\
\hline \multicolumn{8}{|l|}{ Unknown function } \\
\hline transposon protein & Os01g0186900 & & 0 & 3 & 3 & 17.03 & 15.89 \\
\hline expressed protein & Os01g0305200 & AT1G69510 & 1 & 9 & 10 & 4.3 & 9.88 \\
\hline RPGR, putative & Os03g0296200 & & 1 & 1 & 2 & 6.77 & 8.97 \\
\hline expressed protein & Os02g0527200 & AT2G27830 & 2 & 4 & 6 & 5.1 & 8.57 \\
\hline expressed protein & Os02g0601000 & & 4 & 0 & 4 & 4.99 & 6.54 \\
\hline expressed protein & Os06g0133300 & & 4 & 2 & 6 & 4.3 & 4.13 \\
\hline expressed protein & Os01g0138500 & AT2G01260 & 0 & 1 & 1 & 2.88 & 4.13 \\
\hline DUF966 domain containing protein & Os01g0975000 & AT5G59790 & 2 & 3 & 5 & 3.48 & 4.04 \\
\hline cyclase/dehydrase family protein & Os01g0772400 & AT4G17650 & 1 & 3 & 4 & 3.36 & 3.38 \\
\hline expressed protein & Os12g0209700 & AT4G10930 & 2 & 3 & 5 & -2.31 & -2.19 \\
\hline expressed protein & Os11g0307600 & & 2 & 0 & 2 & -7.41 & -2.37 \\
\hline Similar to pnn protein & Os12g0516700 & & 1 & 23 & 24 & -3.88 & -3.67 \\
\hline
\end{tabular}

${ }^{\mathrm{a}}$ Occurrence of GCC-box (GCCGCC or GGCGGC) in $2 \mathrm{~kb}$ upstream region of differentially expressed genes in bbrl mutant compared to wild type.

b Average values of Xoo inoculated bbrl samples, compared to WT samples, from two independent microarray analysis. Numbers show the factor of change between wild type and mutant after Xoo inoculation or no treatment (NT); positive values represent up-regulation (e. g. $3=3$-fold increase), negative values down-regulation (e. g. $-3=3$-fold decrease). 
Table S2. Seventy-nine DRE binding domain containing differentially expressed genes in bbrl mutant compared to wild type.

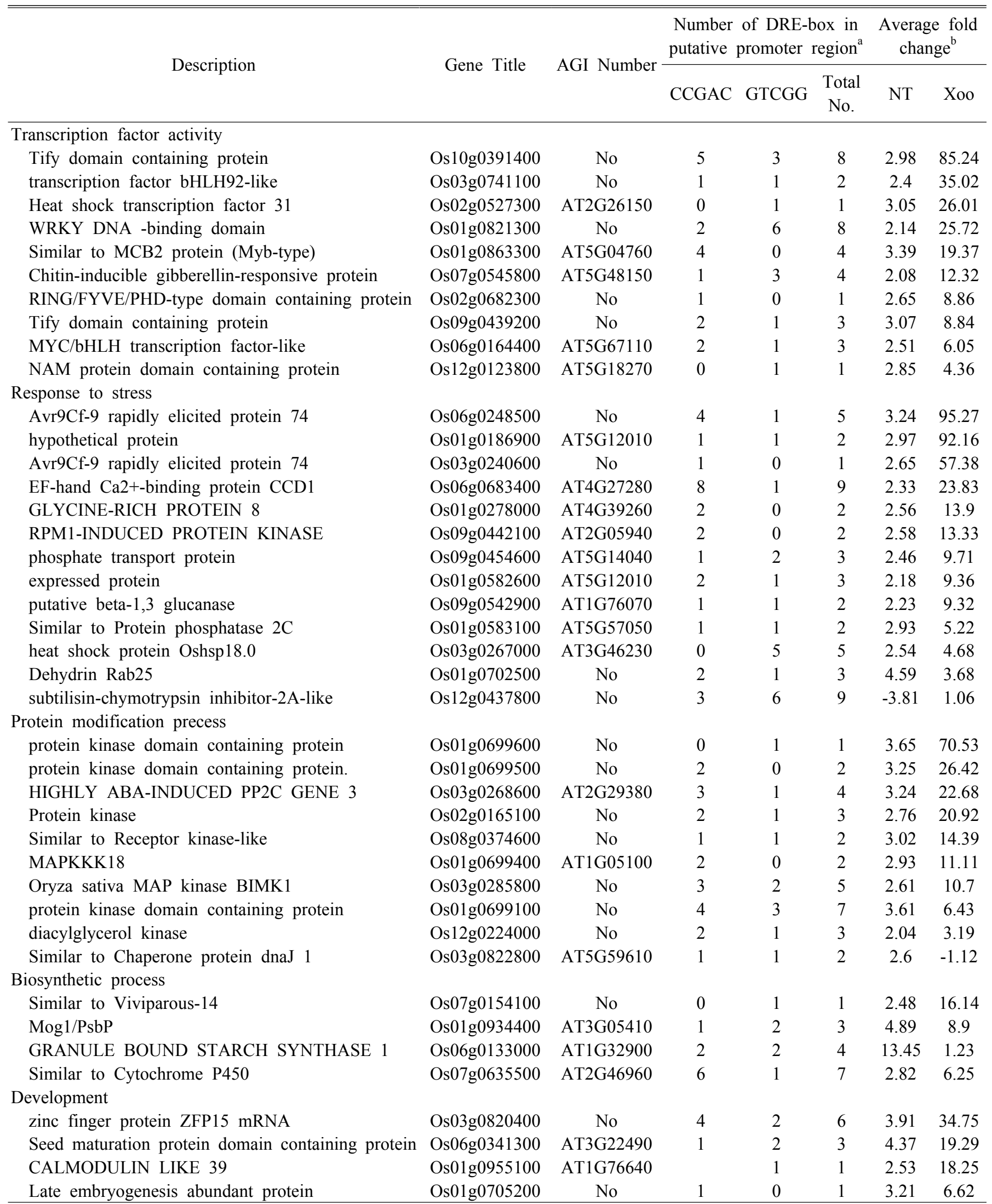


Table S2. Continued.

\begin{tabular}{|c|c|c|c|c|c|c|c|}
\hline \multirow{2}{*}{ Description } & \multirow{2}{*}{ Gene Title } & \multirow{2}{*}{ AGI Number } & \multicolumn{3}{|c|}{$\begin{array}{l}\text { Number of DRE-box in } \\
\text { putative promoter region }\end{array}$} & \multicolumn{2}{|c|}{$\begin{array}{l}\text { Average fold } \\
\text { change }^{b}\end{array}$} \\
\hline & & & CCGAC & GTCGG & $\begin{array}{l}\text { Total } \\
\text { No. }\end{array}$ & NT & Xoo \\
\hline \multicolumn{8}{|l|}{ Gene regulation } \\
\hline arginine/serine-rich 12 & Os12g0516700 & & 1 & 17 & 18 & -3.84 & -3.63 \\
\hline \multicolumn{8}{|l|}{ Metabolic process } \\
\hline Nuclease, Phoaphatase & Os01g0716800 & AT1G71710 & 1 & 2 & 3 & 2.87 & 15.91 \\
\hline$\alpha / \beta$ hydrolase fold- 3 domain containing protein & Os03g0790500 & AT5G06570 & 2 & 0 & 2 & 2.83 & 11.52 \\
\hline putative beta-1,3 glucanase & Os03g0792800 & AT1G64760 & 1 & 2 & 3 & 2.91 & 6.35 \\
\hline putative 4-coumarate-CoA ligase & Os01g0901600 & AT5G63380 & 1 & 1 & 2 & 2.25 & 5.04 \\
\hline putative axi 1 protein & Os01g0851100 & AT2G37980 & 2 & 0 & 2 & 3.62 & 6.9 \\
\hline Anthranilate synthase component I family protein & Os03g0264400 & AT5G05730 & 2 & 2 & 4 & 2.24 & 4.94 \\
\hline dehydrase family protein & Os01g0772400 & AT4G17650 & 2 & 1 & 3 & 4.09 & 2.8 \\
\hline Similar to GTP-binding nuclear protein Ran1B & Os06g0600301 & AT5G55190 & 1 & 0 & 1 & 2.15 & 2.12 \\
\hline \multicolumn{8}{|l|}{ Nucleotide binding } \\
\hline WD-40 repeat family protein & Os01g0383700 & AT4G03020 & 4 & 2 & 6 & 3.56 & 6.49 \\
\hline \multicolumn{8}{|l|}{ Unknown function } \\
\hline ZIM domain containing protein & Os03g0181100 & No & 3 & 5 & 8 & 2.73 & 56.55 \\
\hline hypothetical protein & Os06g0133500 & No & 1 & 0 & 1 & 3.01 & 45.58 \\
\hline hypothetical protein & Os02g0733900 & No & 1 & 1 & 2 & 3.47 & 44.9 \\
\hline hypothetical protein & Os06g0133300 & No & 0 & 1 & 1 & 2.34 & 7.59 \\
\hline DUF604 family protein & Os03g0269900 & AT2G37730 & 1 & 0 & 1 & 3.6 & 7.49 \\
\hline DUF966 family protein & Os01g0975000 & No & 2 & 2 & 4 & 2.24 & 6.31 \\
\hline Cyclin-like F-box domain containing protein & Os07g0561300 & No & 5 & 2 & 7 & 3.9 & 6.17 \\
\hline hypothetical protein & Os09g0445600 & AT2G31940 & 0 & 2 & 2 & 4.22 & 5.75 \\
\hline DUF789 family protein & Os01g0138500 & AT2G01260 & 4 & 4 & 8 & 2.28 & 5.22 \\
\hline TonB box domain containing protein & Os09g0532000 & No & 1 & 0 & 1 & 2.35 & 4.15 \\
\hline Conserved hypothetical protein & Os01g0121500 & No & 1 & 0 & 1 & 2.88 & 3.07 \\
\hline hypothetical protein & Os07g0516400 & No & 1 & 2 & 3 & 4.83 & 3 \\
\hline metallothionein-like type 2 (OsMT-2) mRNA & Os01g0149200 & No & 1 & 3 & 4 & 2.77 & 2.78 \\
\hline hypothetical protein & Os12g0209700 & No & 4 & 2 & 6 & -2.36 & -2.14 \\
\hline Hydroxyproline-rich glycoprotein DZ-HRGP & Os11g0307600 & No & 3 & 0 & 3 & -4.59 & -2.89 \\
\hline similar to GT-2 factor & Os02g0104500 & No & 2 & 0 & 2 & -5.62 & -2.1 \\
\hline hypothetical protein & Os01g0303800 & No & 3 & 3 & 6 & -2.08 & -14.42 \\
\hline
\end{tabular}

${ }^{\mathrm{a}}$ Occurrence of DRE binding domain (CCGAC or GTCGG ) in $2 \mathrm{~kb}$ region up stream of differentially expressed genes in $b b r 1$ mutant compared to wild type.

b Average values of Xoo inoculated $b b r l$ samples, compared to WT samples, from two independent microarray analysis. Numbers show the factor of change between wild type and mutant after Xoo inoculation or no treatment (NT); positive values represent up-regulation (e. g. $3=3$-fold increase), negative values down-regulation (e. g. $-3=3$-fold decrease) 
Table S3. Fifty-one RAV1 binding domain containing differentially expressed genes in bbrl mutant compared to wild type.

\begin{tabular}{|c|c|c|c|c|c|c|c|}
\hline Gene Description & Gene Title & AGI Number & \multicolumn{3}{|c|}{$\begin{array}{c}\text { Number of RAV-binding } \\
\text { site in putative promoter } \\
\text { region }^{\text {a }}\end{array}$} & \multicolumn{2}{|c|}{$\begin{array}{l}\text { Average fold } \\
\text { change }^{b}\end{array}$} \\
\hline \multicolumn{8}{|l|}{ Transcription factor activity } \\
\hline \multicolumn{8}{|l|}{ Response to stress } \\
\hline U-box protein CMPG1 & Os06g0248500 & AT5G37490 & 0 & 1 & 1 & 14.72 & 20.82 \\
\hline cytochrome $\mathrm{P} 450$ & Os12g0150200 & AT2G27690 & 1 & 1 & 2 & 14.57 & 18.57 \\
\hline Similar to ATL31 and ATL6 & Os02g0759400 & AT5G27420 & 3 & 0 & 3 & 9.25 & 12.55 \\
\hline HSF-type DNA-binding domain containing protein & Os02g0527300 & AT5G03720 & 1 & 1 & 2 & 6.84 & 11.51 \\
\hline phosphate carrier protein & Os09g0454600 & AT3G48850 & 1 & 0 & 1 & 3.86 & 6.19 \\
\hline Similar to RPM1-induced kinase & Os09g0442100 & AT2G05940 & 1 & 0 & 1 & 5.76 & 5.96 \\
\hline Similar to PEN3 & Os01g0609300 & AT1G59870 & 1 & 0 & 1 & 5.68 & 5.62 \\
\hline expressed protein & Os01g0582600 & AT5G12010 & 1 & 0 & 1 & 4.24 & 4.81 \\
\hline Similar to $4 \mathrm{CL}$ & Os08g0448000 & AT3G21240 & 0 & 1 & 1 & 4.55 & 4 \\
\hline glutamate decarboxylase & Os03g0236200 & AT5G17330 & 2 & 1 & 3 & 4.27 & 3.46 \\
\hline Similar to PR-6 proteinase inhibitor family & Os12g0437800 & AT2G38870 & 1 & 0 & 1 & -3.81 & 1.06 \\
\hline heat shock protein DnaJ & Os03g0822800 & AT5G59610 & 1 & 0 & 1 & 2.6 & -1.12 \\
\hline \multicolumn{8}{|l|}{ Protein modification process } \\
\hline STE_MEKK_ste11_MAP3K.7 - STE kinases & Os01g0699600 & AT2G32510 & 1 & 2 & 3 & 18.13 & 14.07 \\
\hline \multicolumn{8}{|l|}{ Nucleotide binding } \\
\hline CCHC-type zinc finger & Os03g0659400 & AT5G47430 & 0 & 1 & 1 & 6.23 & 9.25 \\
\hline WD domain & Os01g0383700 & AT4G03020 & 1 & 0 & 1 & 4.42 & 5.19 \\
\hline \multicolumn{8}{|l|}{ Development } \\
\hline EF hand family protein & Os06g0683400 & AT2G46600 & 1 & 0 & 1 & 7.94 & 6.94 \\
\hline growth regulator related protein & Os01g0851100 & AT2G37980 & 1 & 0 & 1 & 3.86 & 6.41 \\
\hline glucan endo-1,3-beta-glucosidase precursor & Os03g0792800 & AT2G19440 & 1 & 0 & 1 & 3.13 & 5.9 \\
\hline senescence-inducible chloroplast stay-green protein 1 & Os09g0532000 & AT4G11910 & 0 & 1 & 1 & 2.72 & 3.57 \\
\hline \multicolumn{8}{|l|}{ Metabolic procss } \\
\hline lumenal PsbP & Os01g0934400 & AT3G05410 & 0 & 2 & 2 & 5.08 & 12.04 \\
\hline expressed protein & Os06g0203600 & AT2G26310 & 1 & 0 & 1 & 9.19 & 11.35 \\
\hline AMP-binding domain containing protein & Os01g0901600 & AT5G63380 & 1 & 0 & 1 & 2.69 & 4.2 \\
\hline \multicolumn{8}{|l|}{ Transport } \\
\hline white-brown complex homolog protein 7 & Os01g0121600 & AT2G01320 & 1 & 2 & 3 & 4.87 & 5.54 \\
\hline Rer1 protein & Os06g0708300 & AT4G39220 & 1 & 0 & 1 & 4.58 & 5.8 \\
\hline ras-related protein & Os06g0600301 & AT5G55190 & 0 & 1 & 1 & 2.17 & 2.06 \\
\hline
\end{tabular}


Table S3. Continued.

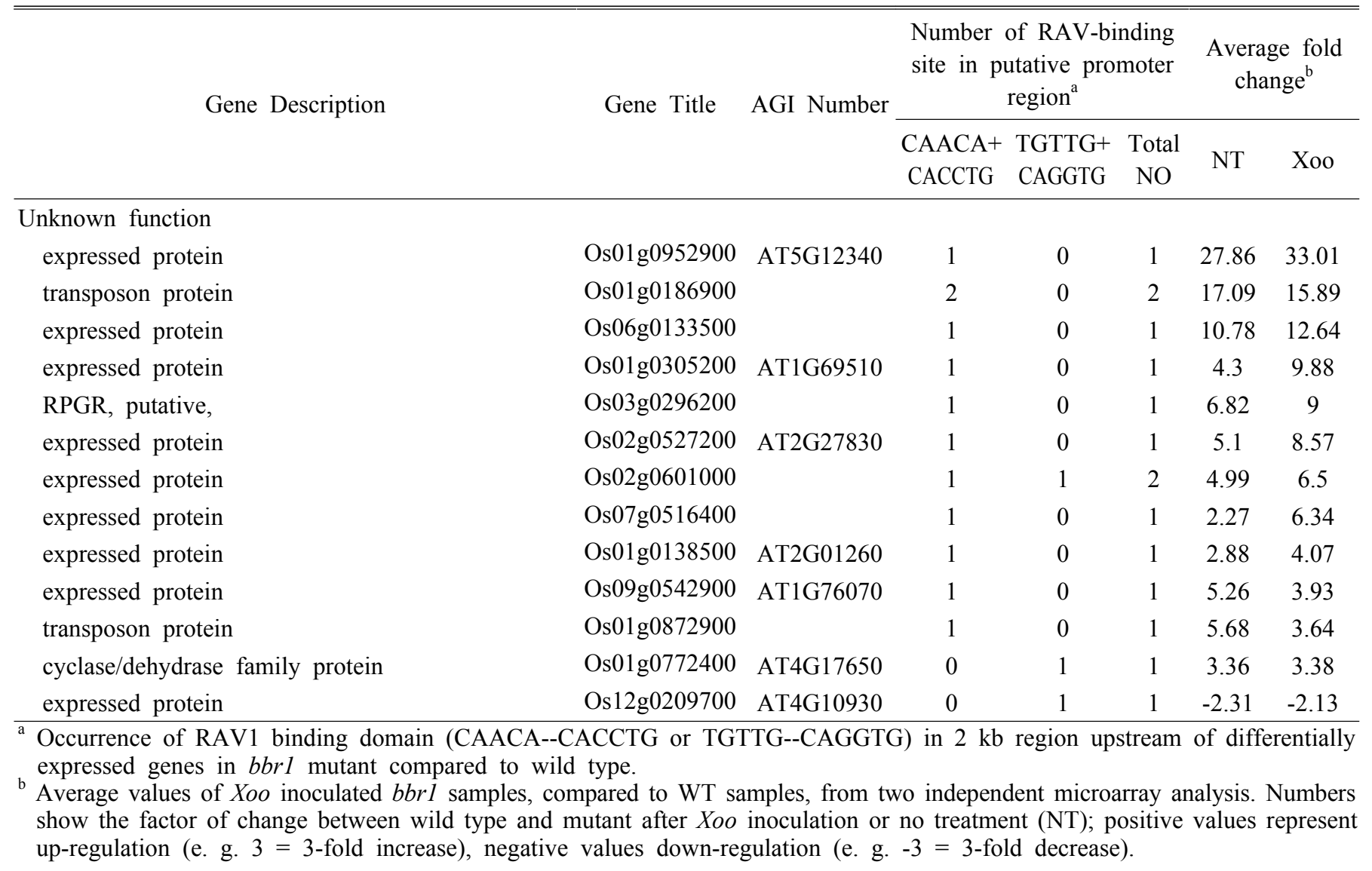

